\title{
Periodontopathogens, Candida spp. and immunological aspects in type 2 diabetes mellitus patients with chronic periodontitis
}

\begin{abstract}
Gabriela Alessandra Da Cruz Galhardo Camargo', Natalia Linhares Coutinho Silva2, Ana Luísa Palhares de Miranda2, Jorge Luiz Mendonça Tributino ${ }^{3}$, Natália Helena Colombo ${ }^{4}$, Cristiane Duque

${ }^{1}$ Department of Periodontology, Fluminense Federal University, Nova Friburgo, Rio de Janeiro, Brazil. Doutor Sylvio Henrique Braune, 22, Centro, 28625-650, Nova Friburgo, Rio de Janeiro, Brazil. Phone number 55- 22-981496066

'LASSBio, Faculty of Pharmacy, Federal University of Rio de Janeiro, Rio de Janeiro, Brazil. Carlos Chagas Filho, 373, CCS, Bloco BSS, sala 22,
\end{abstract} 21941-902, Rio de Janeiro, Brazil. Phone number 55-21-25626503 ${ }^{3}$ Institute of Biomedical Sciences, Federal University of Rio de Janeiro, Rio de Janeiro, Brazil. Carlos Chagas Filho 373, CCS, Bloco J sala J01-029, Ilha do

Fundão, 21941-902, Rio de Janeiro, Brazil. Phone number 55-21-22805694

${ }^{4}$ Department of Pediatric Dentistry and Public Health, Araçatuba Dental School, Univ Estadual Paulista (UNESP), Araçatuba, Brazil. José Bonifácio, 1193, Vila Mendonça, 16015050, Araçatuba, São Paulo, Brazil. Phone number 55- 18-36363315

\begin{abstract}
This study aimed to evaluate clinical, microbiological and immunological parameters in type 2 diabetes mellitus (DM) in comparison with normoglycemic patients (NDM). Glycemic and lipid profiles and periodontal clinical status were determined for thirty-three patients (17 DM and 16 NDM). The presence of periodontopathogens and species of Candida in subgingival sites were determined by polymerase chain reaction and immunological parameters by ELISA assays. All glycemic and clinical parameters evaluated were higher in the DM group, with statistical difference for fasting glucose, glycated-hemoglobin, and periodontal parameters. Lipid profile (except triglycerides), levels of TNF-a and myeloperoxidase and the prevalence of the tested microorganisms were similar between the groups, except for Candida albicans and Candida glabrata, which was higher in the DM group. In conclusion, although microbiological and immunological parameters were similar in the DM and NDM groups, periodontitis and the levels of some species of Candida were more severe in DM patients.
\end{abstract}

Keywords: Diabetes mellitus, periodontal disease, Candida spp., TNF-a, myeloperoxidase.

\section{Introduction}

Received for publication: September 19, 2016 Accepted: April 24, 2017

Correspondence to: Profa. Dra. Cristiane Duque Department of Pediatric Dentistry and Public Health, Araçatuba Dental School, Univ Estadual Paulista (UNESP), Araçatuba, Brazil. José Bonifácio, 1193, Vila Mendonça, 16015050, Araçatuba, São Paulo, Brazil. Phone number 55- 18-36363315 e-mail: cduque@foa.unesp.br, cristianeduque@yahoo.com.br
Type 2 diabetes mellitus (DM) is a metabolic disease characterized by insulin resistance and relative or absolute insulin deficiency ${ }^{1}$. This type of diabetes represents $85-90 \%$ of the diabetic group and is associated with lifestyle factors, mainly obesity and lack of physical activity, and genetic susceptibility. Blood glucose levels can be controlled with dietary changes and body fat reduction ${ }^{2}$. The prevalence of diabetes mellitus has increased significantly and it is estimated that by the year 2030 the world population of people with diabetes will be around 552 million, and in Brazil will reach 19 million $^{3}$.

Patients with chronic hyperglycemia have increased susceptibility to opportunistic infections, as well as oral infections ${ }^{4}$. Periodontitis is the most common chronic oral 
infection, after dental caries, that causes tooth loss in adults and has been considered the sixth most common complication of diabetes mellitus ${ }^{3}$. Periodontal disease is more prevalent and more severe in diabetes mellitus than in normoglycemic patients and periodontal infection may impair glycemic control, since it is able to activate a systemic inflammatory response ${ }^{4}$. Thus, investigators have considered the mechanism of both diseases to be bidirectional, suggesting that one influences the control of the other ${ }^{5}$.

There are conflicting data regarding whether DM individuals with chronic periodontitis present an altered subgingival microbiota compared with nondiabetic patients. Ebersole et al. ${ }^{6}$ reported that periodontitis sites in DM individuals showed a higher frequency of Aggregatibacter actinomycetemcomitans, Porphyromonas gingivalis and Campylobacter spp. when compared with nondiabetic patients. Field et al. ${ }^{7}$ quantified the subgingival levels of Aggregatibacter actinomycetemcomitans, Fusobacterium nucleatum and Porphyromonas gingivalis in patients with periodontitis and found no significant differences between the subgingival microbiota of DM patients compared with non-diabetic subjects. A report demonstrated that the periodontal pockets of DM patients harbored a higher prevalence of Candida spp., mainly $C$. albicans and $C$. dubliniensis, compared with periodontal sites from non-diabetic patients ${ }^{8}$. The same study reported a lower prevalence of Tannerella forsythia in periodontitis sites of DM patients compared with non-diabetic patients ${ }^{8}$.

The presence of bacteria is an essential primary factor for the onset of periodontal disease, however, considering the presence of this alone is not sufficient to predict the onset or severity of periodontal disease, it is also necessary to consider the susceptibility of the host which contributes significantly to the appearance of pathological changes in the periodontium; an imbalance between host and microorganisms that may be caused by local or systemic factors, such as diabetes mellitus, is required ${ }^{8}$.

Studies have pointed out that tissue destruction in periodontal disease results from the interaction of bacteria and their products (such as lipopolysaccharide - LPS) with macrophage activation, triggering the local secretion of inflammatory mediators, especially tumor necrosis factor - $\alpha$ (TNF- $\alpha$ ), interleukin - $1 \beta$ (IL-1 $\beta)$, prostaglandin - E2 (PGE2) and interleukin - 6 (IL-6) $)^{9,10}$. These mediators are responsible for periodontal breakdown, leading to the clinical signs and symptoms of periodontal disease ${ }^{10}$. In diabetes mellitus, these mechanisms seem to be accomplished through a low resistance to infection, exaggerated inflammatory response and a deficiency in healing, justifying the severity of periodontal disease in these patients ${ }^{4}$.

In periodontal disease, neutrophils degranulation can release myeloperoxidase (MPO), among other inflammatory mediators, and MPO has been appointed as a promising marker of periodontal disease activity 11 . In systemically healthy patients, MPO levels were higher in periodontitis sites compared to gingivitis and healthy sites ${ }^{12}$. Gonçalves et al. ${ }^{13}$ analyzed MPO activity in the gingival crevicular fluid of DM with inadequate metabolic control and non-diabetic patients, both with chronic periodontitis, and observed that MPO activity was lower in DM compared to the control group before and after periodontal treatment. Furthermore, after the periodontal therapy the MPO activity was decreased in both groups ${ }^{13}$.

The aim of this study was to evaluate clinical parameters, the presence of putative periodontopathogenic bacteria and Candida spp. and the levels of TNF- $\alpha$ and MPO in subgingival sites of non-diabetic and type 2 diabetes mellitus patients with chronic periodontitis.

\section{Material and methods}

Thirty-three patients ( 17 with type 2 diabetes mellitus (DM) aged $53.41+9.48,16$ non-diabetes mellitus patients (NDM) aged $47.87 \pm 10.37$ were selected to participate in this study. All subjects were recruited from the Department of Periodontology, School of Dentistry, Fluminense Federal University, Nova Friburgo, Rio de Janeiro State, Brazil, over a period of 6 months between 2011 and 2012. The study protocol was approved (protocol number: CAAE - 0434.0.258.000-11) by the Ethics Committee of the School of Medicine, Fluminense Federal University. Prior to participation, the purpose and procedures of the study were fully explained to all patients, who consequently gave written informed consent in accordance with the Helsinki Declaration. Medical and dental histories were taken and patients received clinical evaluation at prescreening visits. The inclusion criteria were: presence of advanced periodontitis defined by bleeding on probing in sites where probing depth was $\geq 5 \mathrm{~mm}$ in a minimum of two teeth in different arches; radiographic bone loss ranging from $30 \%$ to $50 \%{ }^{14,15}$. The exclusion criteria were: patients submitted to periodontal treatment in the last 6 months; patients with systemic diseases; osteoporosis; pregnant lactating females; use of immune suppressive medication, phenytoin, cyclosporine, calcium channel blockers or any use of antibiotics or nonsteroidal anti-inflammatory drugs in the previous 3 months; and any medical conditions requiring immunotherapy or a diagnosis of HIV + or AIDS, which could interfere with the periodontium status.

\section{Glycemic and lipid profile parameters}

A specialized professional collected blood samples from the peripherical vein (cubital fossa) of the individuals who had fasted overnight. Samples were collected in vacuum collection tubes and sent to the Raul Sertã Hospital Laboratory at Nova Friburgo/RJ for clinical analysis of the following glycemic and lipid parameters: fasting glucose levels (FGL), glycosylated hemoglobin (HbA1c), triglycerides (TRG), High-Density Lipoprotein (HDL) and Low-Density Lipoprotein (LDL), using specific kits (Gold Analisa, Belo Horizonte/MG).

\section{Clinical examination}

An experienced periodontist evaluated the clinical parameters and selected two unirradicular teeth for the protocol procedure. Each selected tooth was measured according to the following periodontal parameters: Plaque index (PI), bleeding on probe (BOP), pocket probing depth (PPD), gingival recession 
(GR), clinical attachment level (CAL) using a periodontal probe PCP15 (PCP-UNC15, Hu-Friedy, Chicago, IL); six sites (mesio-buccal, mediobuccal, disto-buccal, mesio-lingual, medio-lingual, disto-lingual ${ }^{16}$ were recorded. Two sites with probing depth $(\mathrm{PPD})>5 \mathrm{~mm}$ were selected for microbiological and immunological analysis. After clinical measurements, the supragingival biofilm was removed with sterile gauze. Gingival crevicular samples were taken from the 4 sites with the deepest PPD ( $\geq 5 \mathrm{~mm}$ ) in each patient, using a sterile paper point from the deepest pocket for 30s. Pooled biofilms from each site were separated in two microtubes containing Tris -EDTA buffer (10 $\mathrm{mM}$ Tris- $\mathrm{HCl}, 0.1 \mathrm{mM}$ EDTA, $\mathrm{pH}$ 7.5) and were stored at $-20^{\circ}$ C. The samples were analyzed microbiologically using PCR.

\section{Microbiological Assessment - PCR assays}

DNA was extracted using a protocol originally described by Sardi et al. ${ }^{8}$, and quantified in a spectrophotometer at 260 nm (Genesys 10UV, Rochester, NY, USA), in order to obtain a standard concentration of $100 \mathrm{ng} / \mathrm{mL}$ and stored at $-20^{\circ} \mathrm{C}$ for subsequent PCR reactions. Briefly, samples were submitted to a lysing solution (extraction buffer and proteinase $\mathrm{K}$ ) and then purified using chloroform: isoamil-alcohol, followed by DNA precipitation with isopropanol and $70 \%$ ethanol. The DNA was ressuspended in TE buffer $(10 \mathrm{mM}$ Tris- $\mathrm{HCl}, 0.1 \mathrm{mM}$ EDTA, pH 7.5, with $10 \mu \mathrm{g} / \mathrm{mL}$ RNAse). Microbial molecular identification was carried out through PCR with specific primers for Aggregatibacter actinomycetemcomitans, Porphyromonas gingivalis, Tannerella forsythia, Prevotella intermedia, Campylobacter rectus, Candida albicans, Candida glabrata, Candida tropicalis and Candida dubliniensis (Table 1). PCR amplification was performed with a TGradient 96 PCR system (TX96, Amplitherm, USA) under thermal conditions specific for each pair of primers. The PCR products were separated by electrophoresis in $2 \%$ agarose gels and Tris-borate-EDTA running buffer ( $\mathrm{pH}$ 8.0). The molecular mass ladder (100 bp DNA ladder, Gibco, Grand Island, NY, USA) was included for running in the agarose gel. The DNA was stained with $0.1 \mu \mathrm{L}$ of Sybr Safe/mL (Invitrogen, CA, USA) and visualized under UV illumination (Pharmacia LKB-MacroVue, San Gabriel, CA, USA). Photographs of the images were taken (Image Mater - LISCAP, VDS, Pharmacia Biotech Piscataway, NJ, USA) and analyzed.

Table 1 - Primers sequences for PCR assays.

\begin{tabular}{|c|c|c|c|}
\hline Species (Reference) & Sequences $\left(5^{\prime}-3^{\prime}\right)$ & Amplicon length(bp) & $\begin{array}{c}\text { Number of accession } \\
\text { GenBank }\end{array}$ \\
\hline $\begin{array}{l}\text { A. actinomycetemcomitans } \\
\text { (Ashimoto et al. }{ }^{17} \text { ) }\end{array}$ & $\begin{array}{l}\text { For: AAA CCC ATC TCT GAC TTC TTC TTC } \\
\text { Rev: ATG CCA ACT TGA CGT TAAT }\end{array}$ & 557 & NC_014629.1 \\
\hline $\begin{array}{c}\text { P. gingivalis } \\
\text { (Benkirane et al. }{ }^{42} \text { ) }\end{array}$ & $\begin{array}{l}\text { For: AAT CGT AAC GGG CGA CAC AC } \\
\text { Rev: GGG TTG CTC CTT CAT CAT AC }\end{array}$ & 593 & NC_002950.2 \\
\hline $\begin{array}{l}\text { T. forsythia } \\
\left(\text { Slots et al. }{ }^{43}\right)\end{array}$ & $\begin{array}{l}\text { For: GCG TAT GTAACC TGC CCG CA } \\
\text { Rev: TGC TTC AGT GTC AGT TAT ACC T }\end{array}$ & 641 & $\begin{array}{l}\text { NC_016610.1 } \\
\text { JAEZ01000014.1 }\end{array}$ \\
\hline $\begin{array}{c}\text { P. intermedia } \\
\text { (Ashimoto et al. }{ }^{17} \text { ) }\end{array}$ & $\begin{array}{l}\text { For: TTT GTT GGG GAG TAAAGC GGG } \\
\text { Rev: TAC ACA TCT CTG TAT CCT GCG T }\end{array}$ & 575 & NC_017861.1 \\
\hline $\begin{array}{c}\text { C. rectus } \\
\text { (Ashimoto et al. }{ }^{17} \text { ) }\end{array}$ & $\begin{array}{l}\text { For: TTTCGGAGCGTAAACTCCTTTTC } \\
\text { Rev: TTTCTGCAAGCAGACACTCTT }\end{array}$ & 595 & ACFU01000050.1 \\
\hline $\begin{array}{l}\text { C. albicans } \\
\text { (Sardi et al. }{ }^{8} \text { ) }\end{array}$ & $\begin{array}{l}\text { For:ACTGCTCAAACCATCTCTGG } \\
\text { Rev: CACAAGGCAAATGAAGGAAT }\end{array}$ & 452 & AJIQ01000008.1 \\
\hline $\begin{array}{l}\text { C. glabrata } \\
\left.\text { (Sardi et al. }{ }^{8}\right)\end{array}$ & $\begin{array}{l}\text { For: GGAGATAGACTGGGCGTTAT } \\
\text { Rev: GTTGTTCAATGGCTTTCTTC }\end{array}$ & 314 & XP_448539.1 \\
\hline $\begin{array}{l}\text { C. tropicalis } \\
\left(\text { Sardi et al. }{ }^{8}\right)\end{array}$ & $\begin{array}{l}\text { For: CACCCAAACAATTACCAAGT } \\
\text { Rev: TGCAAACTCTTTACCTGGAT }\end{array}$ & 253 & NW_003020040.1 \\
\hline $\begin{array}{l}\text { C. dubliniensis } \\
\text { (Donnelly et al. }{ }^{44} \text { ) }\end{array}$ & $\begin{array}{l}\text { For: GTATTTGTCGTTCCCCTTTC } \\
\text { Rev: GTGTTGTGTGCACTAACGTC }\end{array}$ & 288 & NC_012860.1 \\
\hline
\end{tabular}

\section{Tumor necrose factor- $a$ (TNF-a) and Myeloperoxidase (MPO) level measurement}

Gingival crevicular fluid (GCF) was sampled 1 week after clinical examination, by a researcher blinded to the clinical parameters. GCF samples were taken from two different sites labeled as 1 or 2 , in both sites the deepest PPD was $\geq 5 \mathrm{~mm}$ and BOP was chosen for sampling the same patient. The sites were isolated with sterile cotton rolls and dried with an air syringe to eliminate the possibility of contamination with saliva. GCF samples were obtained by placing a calibrated, volumetric microcapillary pipette with an internal diameter of $1.1 \mathrm{~mm}$ and a capacity of $5 \mu \mathrm{L}$. Sites that did not express the appropriate volume of fluid and micropipettes, which were contaminated with blood and saliva, were not included in the study ${ }^{14}$. The GCF was immediately placed into separate tubes containing $250 \mu \mathrm{L}$ phosphate-buffered saline. The samples were stored at $-20^{\circ} \mathrm{C}$ and analyzed by a single, blinded examiner using commercial kits of TNF- $\alpha$ and MPO enzyme-linked immunosorbent assay (R\&D Systems, Minneapolis, MN, USA).

\section{Statistical Analysis}

Required sample size was determined by OpenEpi (OpenEpi, Version 3.03a, 2015) and was calculated to detect a 0.05 difference between BOP (NDM) and BOP (DM) with a power level of $84 \%$. 
The samples size was based on previous studies in the literature ${ }^{18,19}$ using data related to differences in mean and standard deviation values, determining a minimum of 13 patients with type 2 diabetes mellitus required to detect significant differences in clinical and laboratorial variables between groups. The primary efficacy variables were whole-mouth mean BOP (NDM) and BOP (DM). Statistical tests were performed using the software SPSS, version 17.0 (Chicago, IL) to compare differences between the DM and NDM groups. Age and gender were compared between the groups using the Student's t-test. The Mann Whitney U test was performed to compare clinical and immunological parameters (PI, BOP, PPD, GR, CAL, Fasting glucose and HbAlc, LDL, HDL, TRG, TNF- $\alpha$ and MPO). Microbiological analysis of periodontopathogenic bacteria was performed using chi-square tests. Spearman's rho tests were applied to explore correlations between clinical, microbiological and immunological parameters, according to the presence of diabetes mellitus. Statistical significance for all variables was defined at the $5 \%$ level.

\section{Results}

\section{Study subjects}

No significant differences were observed between the DM and NDM groups, considering age (DM: 53.41 \pm 9.48 and NDM: $47.87 \pm 10.37, p=0.751)$ or gender (DM: Male $52.9 \%$, Female 47.55 and NDM: Male $37.5 \%$, Female $62.5 \%, p=0.292)$. The mean duration of diabetes was $7.76 \pm 7.61$ years ranging from 1 to 30 years.

\section{Glycemic and lipid profiles}

Descriptive statistics with means/medians/quartiles of the glycemic and lipid variables are presented in Table 2. Fasting glucose levels and $\mathrm{HbA} 1 \mathrm{c}$ were statistically higher in DM compared to NDM showing glycemic differences between the groups. DM patients had a poorer glycemic control. Positive correlations were found between $\mathrm{HbAlc}$ and BOP in diabetic patients (Spearman's rho, 0.502, p=0.04). LDL and HDL levels were similar between the groups and remained within the normal values. TRG values were statistically higher (Mann Whitney test, $p=0.03$ ) in diabetic compared to non-diabetic patients. TRG was also positively correlated with GR (Spearman's rho, 0.568, $\mathrm{p}=0.017$ ) and CAL (Spearman's rho, 0.516, $\mathrm{p}=0.034$ ).

\section{Periodontal parameters}

PI and BOP indices were evaluated on all tooth surfaces and determined for the whole mouth (\%). PPD, GR and CAL were calculated in millimeters. Means/medians/quartiles of the clinical parameters recorded for both groups are summarized in Table 2. Diabetic patients presented significantly higher values for PD, GR and CAL compared to the control group. The other clinical parameters did not differ significantly between the groups. For both groups, positive correlations (0.491-0.910) were observed between PPD, GR and CAL values, showing that these parameters are intrinsically associated with the periodontal status (Spearman's rho, $\mathrm{p}<0.05)$.
Table 2 - Summary of clinical and laboratorial parameters and proinflammatory mediators levels for DM and NDM groups. Data were expressed in medians (inter-quartiles).

\begin{tabular}{|c|c|c|c|c|c|}
\hline & & & DM & NDM & $p$ value \\
\hline \multirow{5}{*}{ 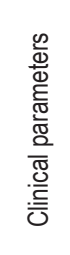 } & & $\mathrm{PI}(\%)^{* *}$ & $69.84(51.78-91.7)$ & 61.94 (43.45-77.94) & 0.363 \\
\hline & & BOP $(\%)$ & $46.66(35.2-65.74)$ & $51.83(24.25-68.34)$ & 0.958 \\
\hline & & PPD (mm) & $5.5(5.24-5.65)$ & $5.19(5-5.29)$ & $0.045^{\star}$ \\
\hline & & $\mathrm{GR}(\mathrm{mm})$ & $1.81(1.33-3.11)$ & $0(0-2.28)$ & $0.045^{*}$ \\
\hline & & $\mathrm{CAL}(\mathrm{mm})$ & $6.1(5.5-7.39)$ & $5.2(5-5,8)$ & $0.014^{*}$ \\
\hline \multirow{5}{*}{ 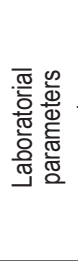 } & $\stackrel{0}{E}$ & $\mathrm{FGL}(\mathrm{mg} / \mathrm{dL})$ & $131(94-172)$ & $89(78.75-97.25)$ & $0.006^{*}$ \\
\hline & 충 & $\mathrm{HbA} 1 \mathrm{c}(\%)$ & $7.6(6.85-8.3)$ & $4.7(4.5-5)$ & $0.000^{*}$ \\
\hline & & $\mathrm{LDL}(\mathrm{mg} / \mathrm{dL})$ & 125 (107-139) & $93(87.5-136.0)$ & 0.165 \\
\hline & 응한 & $\mathrm{HDL}(\mathrm{mg} / \mathrm{dL})$ & 44 (38-48) & $47.5(40.75-55.5)$ & 0.102 \\
\hline & & TGR (mg/dL) & $146(89-261)$ & $74(54.5-154)$ & $0.033^{*}$ \\
\hline \multirow{2}{*}{ 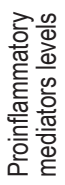 } & & $\begin{array}{l}\text { TNF-a (pg/ } \\
\mathrm{mL})\end{array}$ & $1.03(0.76-1.22)$ & $1.18(0.9-1.49)$ & 0.068 \\
\hline & & $\begin{array}{c}\text { MPO (pg/ } \\
\mathrm{mL})\end{array}$ & $0.028(0.01-0.07)$ & $0.06(0.026-0.079)$ & 0.382 \\
\hline
\end{tabular}

* Statistically significant difference between DM and NDM groups (Mann Whitney U-test, $p<0.05$ ).

${ }^{* *}$ Plaque index $(\mathrm{PI})$, bleeding on probe (BOP), pocket probing depth (PPD), gingival recession (GR), clinical attachment level (CAL), fasting glucose levels (FGL) glycosylated hemoglobin (HbA1c), triglycerides (TRG), High -Density Lipoprotein (HDL) and Low-Density Lipoprotein (LDL).

\section{Microbiological analysis}

Figure 1 shows the prevalence of $A$. actinomycetemcomitans (Aa), $P$. gingivalis $(\mathrm{Pg}), T$. forsythia $(\mathrm{Tf}), P$. intermedia $(\mathrm{Pi}), C$. rectus $(\mathrm{Cr}), C$. albicans $(\mathrm{Ca}), C$. glabrata $(\mathrm{Cg}), C$. tropicalis $(\mathrm{Ct})$ and $C$. dubliniensis $(\mathrm{Cd})$ in the DM and NDM groups. A significant difference between the groups was observed only for $C$. albicans and $C$. glabrata. The prevalence of putative periodontopathogens (Aa, Pg, Tf, Pi and $\mathrm{Cr}$ ) was similar between DM and NDM patients. Twenty-four of the 33 patients (17 DM and 7 NDM) were colonized by $C$. albicans and twenty (13 DM and 7 NDM) by $C$. glabrata. All patients harbored $C$. dubliniensis and none presented $C$. tropicalis. The percentage of sites harboring $C$. albicans, $T$. forsythensis and $P$. gingivalis simultaneously was statistically higher in DM compared to NDM patients (Figure 2). The association of four or five periodontopathogens and $C$. albicans or C. glabrata was observed only in DM patients (Figures 2 and 3).

\section{Immunological analysis}

Levels of TNF- $\alpha$ and MPO measured in gingival crevicular fluid are presented in Table 2. No statistically significant differences were found for either proinflammatory marker between the DM and NDM groups. No correlations were verified between these biological markers and clinical, glycemic, lipid or microbiological profiles (Spearman's rho correlation, $\mathrm{p}>0.05$ ). 


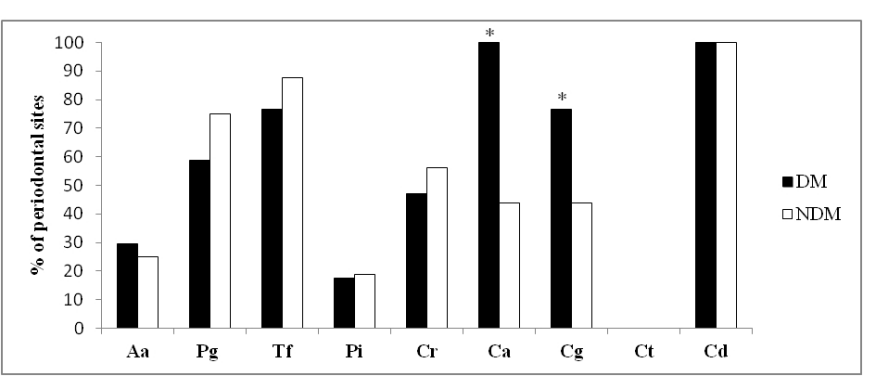

Fig. 1 - Frequency of periodontal sites harboring Aggregatibacter actinomycetemcomitans (Aa), Porphyromonas gingivalis (Pg), Tannerella forsythia (Tf), Prevotella intermedia (Pi), Campylobacter rectus (Cr), Candida albicans $(\mathrm{Ca})$, Candida glabrata $(\mathrm{Cg})$, Candida tropicalis $(\mathrm{Ct})$ and Candida dubliniensis $(\mathrm{Cd})$ in the DM and NDM groups.

* Statistically significant differences were found between the groups for $\mathrm{Ca}$ and $\mathrm{Cg}$, according to $\chi^{2}$ tests (test, $\mathrm{p}<0.05$ ).

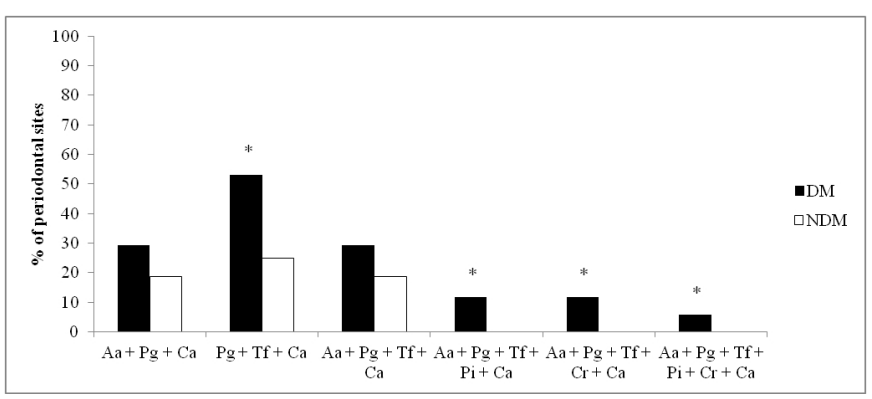

Fig. 2 - Frequency of periodontal sites (\%) harboring combinations of putative periodontopathogens with Candida albicans $(\mathrm{Ca})$ in the DM and NDM groups. *Statistically significant differences between the DM and NDM groups, according to $\chi 2$ tests $(\mathrm{p}<0.05)$. Aggregatibacter actinomycetemcomitans (Aa), Porphyromonas gingivalis (Pg), Tannerella forsythia (Tf), Prevotella intermedia, Campylobacter rectus (Cr).

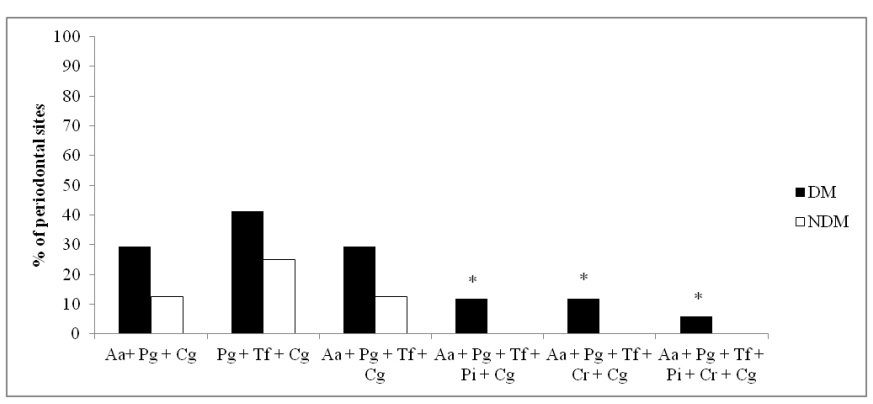

Fig. 3 - Frequency of periodontal sites (\%) harboring combinations of putative periodontopathogens with Candida glabrata $(\mathrm{Cg})$ in the DM and NDM groups. *Statistically significant differences between the DM and NDM groups, according to $\chi 2$ tests $(\mathrm{p}<0.05)$. Aggregatibacter actinomycetemcomitans (Aa), Porphyromonas gingivalis (Pg), Tannerella forsythia (Tf), Prevotella intermedia, Campylobacter rectus (Cr).

\section{Discussion}

Although the majority of the published studies are crosssectional, providing a limited possibility of a causal-effect relationship ${ }^{10}$, substantial evidence has indicated diabetes mellitus $(\mathrm{DM})$ as a risk factor for periodontal disease $(\mathrm{PD})^{3}$. The results of the present study confirmed that patients with type 2 diabetes mellitus (DM) presented significantly higher values of PPD, GR and CAL compared to the control group and consequently greater severity of PD. The 2009-meta-analysis, investigated whether or not diabetes is a risk factor for periodontitis and included papers published between 1980 and 2007. Twenty-seven crosssectional studies were included and detected more periodontal disease in diabetic compared to non-diabetic subjects. Although methodological flaws were found, the results showed a significant association between CAL (difference 1.00, 95\% CI 0.15-1.84), PPD (difference 0.46, 95\% CI 0.01-0.91) and DM, confirming that $\mathrm{DM}$ is an important risk factor for periodontitis ${ }^{5}$.

Glycemic profile is usually measured in studies with diabetics to evaluate the influence of these parameters on the prevalence, extent and severity of periodontitis. Generally, well-controlled diabetes with glycemic parameters within the normal values seems to have little effect on the risk of periodontitis, however, the contrary is observed in poorly controlled patients. In the current study, fasting glucose levels and $\mathrm{HbAlc}$ were statistically higher in DM compared to NDM patients. Our glycemic results are consistent with important systematic reviews that investigated associations between diabetes, glycemic control and complications, showing that PD was more prevalent among individuals with poorer glycemic control ${ }^{5,20}$. Both studies supported the evidence that diabetes has an adverse effect on periodontal status and PD has an adverse effect on glycemic control. Due to the crosssectional design of the present study, it is not possible to state that periodontal disease had an effect on glycemic control. This evidence could be provided by treatment and longitudinal studies. In poorly controlled diabetes, there is a nonspecific glycation of lipids and proteins that forms reactive oxygen species (ROS). In the case of DM, ROS are considered as a major risk for developing micro and macrovascular complications. They participate in the formation of advanced glycated end products (AGE) that induce crosslinkation processes in the structure of proteins, such as collagen, modifying blood vessel structure. After binding to their specific receptors (RAGE), they lead to cytokine production and proinflammatory effects ${ }^{21}$. All these factors can exacerbate the severity of periodontal disease, besides contributing to the development of systemic complications such as retinopathy, nephropathy, neuropathy and other diseases ${ }^{4}$.

Another occurrence concomitant to diabetes mellitus is dyslipidemia, defined as a high blood concentration of lipids, especially LDL and triglycerides (TRG). Studies have indicated an association between elevation in blood lipoproteins and alterations in periodontal disease $\mathrm{e}^{2,22}$. However, other clinical trials failed to identify this relationship ${ }^{22,23}$. In the current study, both groups with periodontal disease, independent of the presence of diabetes, presented normal values of LDL. However, DM patients had high levels of TRG, above normal values ( $>150 \mathrm{mg} / \mathrm{dL}$ ), and were positively correlated with two important periodontal parameters, GR and CAL. Our results are in agreement with Tu et al. ${ }^{24}$ who evaluated associations between lipid parameters and periodontitis and found a positive correlation for TRG and C-reactive protein (CRP). Controversially, Sora et al. ${ }^{25}$ evaluated the relationship of metabolic syndrome (five cardiovascular risk factors: abdominal obesity, hypertension, reduced HDL, elevated TRG and elevated 
fasting glucose or diabetes), and these factors individually, with the extent of severe periodontitis among patients with DM from Gullah. They found a relationship between metabolic syndrome and periodontitis in this population; however, no results from the multivariable binomial regression demonstrated a relationship between the isolated components of metabolic syndrome, including HDL and TRG, and periodontitis in these individuals with diabetes. The authors explained that more than one risk factor linked to diabetes is necessary to increase the extent of periodontitis and commented that their results may not apply to other populations with a different genetic and socio-cultural background. However, Almeida Abdo et al. ${ }^{26}$ found no associations between dyslipidemia and periodontal disease. They observed that diabetes, age and smoking had positive correlations with periodontitis $(\mathrm{CAL} \geq 3$ and $\mathrm{CAL} \geq 5$ ).

Periodontal health is dependent on a balance between the bacteria harboring the subgingival biofilm and the host response to them. Environmental changes may modify the bacterial challenge or host immune response, as occurs in diabetes patients ${ }^{7}$. The prevalence of putative periodontal bacteria in DM compared to NDM has been previously investigated by some authors $7,8,27,28$. The bacterial species belonging to the "red and orange complexes" are the most frequently studied and there is no consensus whether specific periodontal pathogens can harbor subgingival sites in subjects with DM when compared with NDM. In the present study, the frequency of periodontal sites harboring $A$. actinomycetemcomitans, $P$. gingivalis, $T$. forsythia, $P$. intermedia and $C$. rectus $(\mathrm{Cr})$ was statistically similar between the groups. Our results corroborate with those found by Field et al. ${ }^{7}$, Sardi et al. ${ }^{8}$ and Yuan et al. ${ }^{28}$ and differ from other studies $^{29-32}$. We focused on subgingival sites with periodontal disease, specifically deep pockets, for both groups, also reported by the majority of these studies. Contrarily, the study conducted by Aemaimanan et al. ${ }^{30}$ found high levels of $\mathrm{T}$. forsythia in healthy sites and $P$. gingivalis and $T$. forsythia in gingivitis sites and a higher quantity of $P$. gingivalis only in periodontal sites of poorly controlled DM patients compared to the NDM group. Recent studies have indicated a high prevalence of $T$. forsythia in DM patients with periodontitis and their possible role in the severity of periodontitis ${ }^{29,30}$. Li et al. ${ }^{31}$ also demonstrated not only higher levels of $T$. forsythia but also $T$. denticola, however lower levels of $P$. intermedia in the subgingival plaque of chinese patients with type 2 diabetes mellitus. Zhou et al. ${ }^{32}$ concluded that subjects with healthy periodontium harbored different genera (abundance of Prevotella, Pseudomonas and Tannerella) compared to patients with periodontitis (abundance in Actinobacteria, Proteobacteria and Bacteriodetes), and in both groups, the authors detected that diabetic and nondiabetic subjects harbored bacteria at several taxonomic levels with significant different prevalence or abundance. These discrepancies in the results could be related to the differences in the lifestyle of the populations, which interferes directly in the composition of the subgingival microbiota ${ }^{33}$.

Diabetic patients are known for their predisposition to oral infections caused by Candida spp., particularly those with poor glycemic control. This condition may occur concomitantly with a higher incidence and greater severity of periodontitis ${ }^{34}$. In the current study, species of Candida were detected from deep pockets in both groups with chronic periodontitis, regardless of the presence of diabetes. However, two of these species, $C$. albicans and $C$. glabrata, were more frequently found in DM patients. Candida spp. have also been identified in periodontal sites by other investigators ${ }^{8,35,36}$, but only the study developed by Melton et al. ${ }^{36}$ demonstrated higher levels of $C$. albicans (53\%) followed by C. glabrata (20\%), similar to those obtained in the present study. $C$. albicans has been described as the most prevalent and pathogenic species of the genus ${ }^{34}$ and plays a role in immune evasion and adherence to epithelium, causing inflammatory reactions ${ }^{37}$ and may be detrimental to the periodontal environment. C. glabrata has emerged as an important opportunist pathogen in oropharyngeal candidiasis, after $C$. albicans. The frequency of C. glabrata infections increased up to $50 \%$ in some populations, due to immunosuppressive therapies and broad spectrum antibiotics ${ }^{37}$. A significant correlation between poor glycemic control and periodontal Candida colonization has been reported ${ }^{8,34,35}$ and is in agreement with our results. Glucose level concentration in gingival crevicular fluid is known to be associated with blood glucose level ${ }^{9}$ and could partly explain the proliferation of Candida in periodontal sites ${ }^{35}$.

Another suggested effect of poorly controlled glycemic status in DM is the aggregation of local cytokine response ${ }^{9}$. In the current study, TNF-alpha was chosen for its intrinsic relationship with the severity of periodontal disease, but this study aimed to evaluate if this cytokine could be more elevated in diabetic patients due to their glycemic status. However, there was no difference between the groups. The relevance of the cytokine profile in subjects with DM and chronic periodontitis has been explored and the levels of these inflammatory markers seem not to differ from normoglycemic patients with a similar periodontal condition ${ }^{9,38}$. Javed et al. ${ }^{38}$ evaluated databases from 1988 to 2011 and concluded that gingival crevicular fluid cytokine profile, including TNF-alpha, in patients with or without DM seems to be related to the severity of periodontal inflammation and the diabetes is secondary. Besides, the action of many of the biomarkers is short-lived and is likely to vary based upon stimulatory molecules, leading to a limited local response at affected sites. After activation of downstream biomarkers and/ or local cell activation, many biomarkers, such as IL-6 and TNF-alpha, are degraded quickly ${ }^{39}$. The myeloperoxidase (MPO) activity was also evaluated in the present study. MPO is one of the peroxidase systems responsible for immunological defense in saliva and gingival crevicular fluid. Studies have demonstrated an increase in the MPO level in systemically healthy patients with periodontitis ${ }^{40}$. For diabetic subjects, two studies showed different results, with greater MPO activity ${ }^{13,41}$. In our study, there was no statistical difference in the MPO levels obtained from gingival crevicular fluid between diabetic and non-diabetic individuals, both with periodontal disease. The presence of diabetes did not influence the level of this proinflammatory marker, in agreement with Tenovuo et al. ${ }^{41}$. Controversially, Gonçalves et al. ${ }^{13}$ found lower MPO activity in diabetic patients with chronic periodontitis, before and after periodontal treatment. There are methodological differences between the present study and the above-mentioned investigations. The first one ${ }^{41}$ analyzed 
saliva samples from patients with different types of diabetes, not exclusively DM and the last one ${ }^{13}$ evaluated enzyme activity, not its concentration, as in the present study. These methodological differences make it difficult to compare the present study with the other investigations.

Within the limitations of this study, mainly the small sample size, it may be concluded that although chronic periodontitis was clinically more severe, the level of TNF- $\alpha$, MPO and the prevalence of putative periodontal pathogens were not different in DM patients compared to non-diabetic individuals. An interesting fact was the high frequency of $C$. albicans and C. glabrata, which seem to have a preferential capacity for colonizing the periodontal pockets of diabetic patients. More longitudinal studies are necessary to confirm the influence of the species of Candida on the progression of periodontal disease.

\section{Acknowledgements}

This study was supported by the Fundação de Amparo a Pesquisa (FAPERJ), Rio de Janeiro, Brazil (grant \# E-26/100.491/2010).

\section{References}

1. American Diabetes Association. Diagnosis and classification of diabetes mellitus. Diabetes Care. 2007 Jan;30 Suppl 1:S42-47.

2. Fentoğlu $\mathrm{O}, \mathrm{Oz} \mathrm{G}$, Taşdelen $\mathrm{P}, \mathrm{U}$ skun E, Aykaç Y, Bozkurt FY. Periodontal status in subjects with hyperlipidemia. J Periodontol. 2009 Feb;80(2):26773. doi: 10.1902/jop.2009.080104.

3. Whiting DR, Guariguata L, Weil C, Shaw J. IDF diabetes atlas: global estimates of the prevalence of diabetes for 2011 and 2030. 2011 Dec;94(3):311-21. doi: 10.1016/j.diabres.2011.10.029.

4. Lalla E, Lamster IB, Drury S, Fu C, Schmidt AM. Hyperglycemia, glycoxidation and receptor for advanced glycation endproducts: potential mechanisms underlying diabetic complications, including diabetesassociated periodontitis. Periodontol 2000. 2000 Jun;23:50-62.

5. Chávarry NG, Vettore MV, Sansone $C$, Sheiham A. The relationship between diabetes mellitus and destructive periodontal disease: a metaanalysis. Oral Health Prev Dent. 2009;7(2):107-27.

6. Ebersole JL, Holt SC, Hansard R, Novak MJ. Microbiologic and immunologic characteristics of periodontal disease in Hispanic Americans with type 2 diabetes. J Periodontol. 2008 Apr;79(4):637-46. doi: 10.1902/ jop.2008.070455.

7. Field CA, Gidley MD, Preshaw PM, Jakubovics N. Investigation and quantification of key periodontal pathogens in patients with type 2 diabetes. J Periodontal Res. 2012 Aug;47(4):470-8. doi: 10.1111/j.16000765.2011.01455.x.

8. Sardi JC, Duque C, Camargo GA, Hofling JF, Gonçalves RB. Periodontal conditions and prevalence of putative periodontopathogens and Candida spp. In insulin-dependent type 2 diabetic and non-diabetic patients with chronic periodontitis--a pilot study. Arch Oral Biol. 2011 Oct;56(10):1098105. doi: 10.1016/j.archoralbio.2011.03.017.

9. Salvi GE, Beck JD, Offenbacher S. PGE2, IL-1 beta, and TNF-alpha responses in diabetics as modifiers of periodontal disease expression. Ann Periodontol. 1998 Jul;3(1):40-50.

10. Preshaw PM, Alba AL, Herrera D, Jepsen S, Konstantinidis A, Makrilakis $\mathrm{K}$, et al. Periodontitis and diabetes: a two-way relationship. Diabetologia. 2012 Jan;55(1):21-31. doi: 10.1007/s00125-011-2342-y.

11. Buduneli N, Kinane DF. Host-derived diagnostic markers related to soft tissue destruction and bone degradation in periodontitis. J Clin Periodontol.
2011 Mar;38 Suppl 11:85-105. doi: 10.1111/j.1600-051X.2010.01670.x.

12. Leppilahti JM, Hernández-Ríos PA, Gamonal JA, Tervahartiala T, Brignardello-Petersen R, Mantyla $P$, et al. Matrix metalloproteinases and myeloperoxidase in gingival crevicular fluid provide site-specific diagnostic value for chronic periodontitis. J Clin Periodontol 2014 Apr;41(4):348-56. doi: $10.1111 /$ jcpe. 12223 .

13. Gonçalves D, Correa FO, Khalil NM, de Faria Oliveira OM, Orrico SR. The effect of non-surgical periodontal therapy on peroxidase activity in diabetic patients: a case-control pilot study. J Clin Periodontol. 2008 Sep;35(9):799806. doi: 10.1111/j.1600-051X.2008.01289.x.

14. Page RC, Eke PI. Case definitions for use in population-based surveillance of periodontitis. J Periodontol. 2007 Jul;78(7 Suppl):1387-99.

15. Camargo GA, Lima MA, Fortes TV, de Souza CS, de Jesus AM, de Almeida RP. Effect of periodontal therapy on metabolic control and levels of IL-6 in the gingival crevicular fluid in type 2 diabetes mellitus. Indian J Dent Res. 2013 Jan-Feb;24(1):110-6. doi: 10.4103/0970-9290.114953.

16. Ainamo J, Bay I. Problems and proposals for recording gingivitis and plaque. Int Dent J. 1975 Dec;25(4):229-35.

17. Ashimoto A, Chen C, Bakker I, Slots J. Polymerase chain reaction detection of 8 putative periodontal pathogens in subgingival plaque of gingivitis and advanced periodontitis lesions. Oral Microbiol Immunol. 1996 Aug;11(4):266-73.

18. Correa FO, Gonçalves D, Figueredo CM, Bastos AS, Gustafsson A, Orrico SR. Effect of periodontal treatment on metabolic control, systemic inflammation and cytokines in patients with type 2 diabetes. J Clin Periodontol. 2010 Jan;37(1):53-8. doi: 10.1111/j.1600-051X.2009.01498.x.

19. Abbass MM, Korany NS, Salama AH, Dmytryk JJ, Safiejko-Mroczka B. The relationship between receptor for advanced glycation end products expression and the severity of periodontal disease in the gingiva of diabetic and non diabetic periodontitis patients. Arch Oral Biol. 2012 Oct:57(10):1342-54. doi: 10.1016/j.archoralbio.2012.06.007.

20. Garcia D, Tarima S, Okunseri C. Periodontitis and glycemic control in diabetes: NHANES 2009 to 2012. J Periodontol. 2015 Apr;86(4):499-506. doi: 10.1902/jop.2014.140364.

21. Radoi V, Lixandru D, Mohora M, Virgolici B. Advance glycation and products in diabetes mellitus: mechanism of action and focused treatment. Proc Rom Acad. 2012;1:9-19.

22. Machado AC, Quirino MR, Nascimento LF. Relation between chronic periodontal disease and plasmatic levels of triglycerides, total cholesterol and fractions. Braz Oral Res. 2005 Oct-Dec;19(4):284-9.

23. Banihashemrad SA, Moeintaghavi A, RafighdoostA. Relationship between cholesterol and triglyceride blood values and periodontal parameters in patients of Mashhad health center. N Y State Dent J. 2008 AugSep;74(5):65-6.

24. Tu YK, D'Aiuto F, Lin HJ, Chen YW, Chien KL. Relationship between metabolic syndrome and diagnoses of periodontal diseases among participants in a large Taiwanese cohort. J Clin Periodontol. 2013 Nov;40(11):994-1000. doi: 10.1111/jcpe.12157.

25. Sora ND, Marlow NM, Bandyopadhyay D, Leite RS, Slate EH, Fernandes JK. Metabolic syndrome and periodontitis in Gullah African Americans with type 2 diabetes mellitus. J Clin Periodontol. 2013 Jun;40(6):599-606. doi: 10.1111/jcpe.12104.

26. Almeida Abdo J, Cirano FR, Casati MZ, Giampaoli V, Viana Casarin RC, Pimentel SP. Influence of dyslipidemia and diabetes mellitus on chronic periodontal disease. J Periodontol. 2013 Oct;84(10):1401-8. doi: 10.1902/ jop.2012.120366.

27. Casarin RC, Barbagallo A, Meulman T, Santos VR, Sallum EA, Nociti FH, et al. Subgingival biodiversity in subjects with uncontrolled type-2 diabetes and chronic periodontitis. J Periodontal Res. 2013 Feb;48(1):30-6. doi: 10.1111/j.1600-0765.2012.01498.x.

28. Yuan K, Chang CJ, Hsu PC, Sun HS, Tseng CC, Wang JR. Detection of putative periodontal pathogens in non-insulin-dependent diabetes mellitus and non-diabetes mellitus by polymerase chain reaction. J Periodontal 
Res. 2001 Feb;36(1):18-24.

29. Hintao J, Teanpaisan R, Chongsuvivatwong V, Ratarasan C, Dahlen G. The microbiological profiles of saliva, supragingival and subgingival plaque and dental caries in adults with and without type 2 diabetes mellitus. Oral Microbiol Immunol. 2007 Jun;22(3):175-81.

30. Aemaimanan P, Amimanan P, Taweechaisupapong S. Quantification of key periodontal pathogens in insulin-dependent type 2 diabetic and nondiabetic patients with generalized chronic periodontitis. Anaerobe. 2013 Aug;22:64-8. doi: 10.1016/j.anaerobe.2013.06.010.

31. Li C, Liu J, Tan L, Yu N, Lin L, Geng F, et al. The sociodemographic characteristics, periodontal health status, and subgingival microbiota of patients with chronic periodontitis and type 2 diabetes mellitus: a casecontrol study in a Chinese population. J Periodontol. 2013 Aug;84(8):105866. doi: 10.1902/jop.2012.120282.

32. Zhou M1, Rong R, Munro D, Zhu C, Gao X, Zhang Q, et al. Investigation of the effect of type 2 diabetes mellitus on subgingival plaque microbiota by high-throughput 16S rDNA pyrosequencing. PLoS One. $2013 \mathrm{Apr}$ 22;8(4):e61516. doi: 10.1371/journal.pone.0061516.

33. Haffajee AD, Bogren A, Hasturk H, Feres M, Lopez NJ, Socransky SS. Subgingival microbiota of chronic periodontitis subjects from different geographic locations. J Clin Periodontol. 2004 Nov;31(11):996-1002.

34. Javed F, Klingspor L, Sundin U, Altamash M, Klinge B, Engström PE. Periodontal conditions, oral Candida albicans and salivary proteins in type 2 diabetic subjects with emphasis on gender. BMC Oral Health. 2009 May 12;9:12. doi: 10.1186/1472-6831-9-12.

35. Hammad MM, Darwazeh AM, Idrees MM. The effect of glycemic control on Candida colonization of the tongue and the subgingival plaque in patients with type II diabetes and periodontitis. Oral Surg Oral Med Oral Pathol Oral Radiol. 2013 Sep;116(3):321-6. doi: 10.1016/j.0000.2013.05.013.

36. Melton JJ, Redding SW, Kirkpatrick WR, Reasner CA, Ocampo GL, Venkatesh A, et al. Recovery of Candida dublinienses and other Candida species from the oral cavity of subjects with periodontitis who had well- controlled and poorly controlled type 2 diabetes: a pilot study. Spec Care Dent. 2010 Nov-Dec;30(6):230-4. doi: 10.1111/j.1754-4505.2010.00159.x.

37. Fidel PL Jr, Vazquez JA, Sobel JD. Candida glabrata: review of epidemiology, pathogenesis, and clinical disease with comparison to $C$. albicans. Clin Microbiol Rev. 1999 Jan;12(1):80-96.

38. Javed F, Al-Askar M, Al-Hezaimi K. Cytokine profile in the gingival crevicular fluid of periodontitis patients with and without type 2 diabetes: a literature review. J Periodontol. 2012 Feb;83(2):156-61. doi: 10.1902/ jop.2011.110207.

39. Geisinger ML, Michalowicz BS, Hou W, Schoenfeld E, Gelato M, Engebretson SP, et al. Systemic Inflammatory Biomarkers and Their Association With Periodontal and Diabetes-Related Factors in the Diabetes and Periodontal Therapy Trial, A Randomized Controlled Trial. J Periodontol. 2016 Aug;87(8):900-13. doi: 10.1902/jop.2016.150727.

40. Yamalik N, Cağlayan F, Kilinç K, Kilinç $A$, Tümer $C$. The importance of data presentation regarding gingival crevicular fluid myeloperoxidase and elastase-like activity in periodontal disease and health status. J Periodontol. 2000 Mar;71(3):460-7.

41. Tenovuo J, Lehtonen OP, Viikari J, Larjava H, Vilja P, Tuohimaa P. Immunoglobulins and innate antimicrobial factors in whole saliva of patients with insulin-dependent diabetes mellitus. J Dent Res. 1986 Jan;65(1):62-6.

42. Benkirane RM, Guillot E, Mouton C. Immunomagnetic PCR and DNA probe for detection and identification of Porphyromonas gingivalis. J Clin Microbiol 1995;33(11):2908-12.

43. Slots J, Ashimoto A, Flynn MJ, Li G, Chen C. Detection of putative periodontal pathogens in subgingival specimens by $16 \mathrm{~S}$ ribosomal DNA amplification with the polymerase chain reaction. Clin Infect Dis 1995;20(Suppl):S304-7.

44. Donnelly SM, Sullivan DJ, Shanley DB, Coleman DC. Phylogenetic analysis and rapid identification of Candida dubliniensis based on analysis of ACT1 intron and exon sequences. Microbiology 1999;145(Pt 8):1871182. 